\title{
Risk Factors of New Cerebral Infarctions After Endovascular Treatment for Basilar Artery Stenosis Based on High-Resolution Magnetic Resonance Imaging
}

\author{
Jichang Luo ${ }^{1,2}$, Long $\mathrm{Li}^{1,2}$, Tao Wang ${ }^{1,2}$, Kun Yang ${ }^{3}$, Yao Feng ${ }^{1,2}$, Renjie Yang ${ }^{1,2}$, Yan Ma ${ }^{1,2}$, \\ Peng Gao ${ }^{1,2,4}$, Bin Yang ${ }^{1,2}$ and Liqun Jiao ${ }^{1,2,4 *}$ \\ ${ }^{1}$ China International Neuroscience Institute (China-INI), Beijing, China, ${ }^{2}$ Department of Neurosurgery, Xuanwu Hospital, \\ Capital Medical University, Beijing, China, ${ }^{3}$ Department of Evidence-Based Medicine, Xuanwu Hospital, Capital Medical \\ University, Beijing, China, ${ }^{4}$ Department of Interventional Neuroradiology, Xuanwu Hospital, Capital Medical University, Beijing, \\ China
}

OPEN ACCESS

Edited by:

Xinyi Leng,

The Chinese University of

Hong Kong, China

Reviewed by:

Wenjie Yang,

Johns Hopkins University,

United States

Edgar A. Samaniego,

The University of lowa, United States

${ }^{*}$ Correspondence:

Liqun Jiao

liqunjiao@sina.cn

Specialty section:

This article was submitted to

Endovascular and Interventional

Neurology,

a section of the journal

Frontiers in Neurology

Received: 21 October 2020 Accepted: 18 December 2020 Published: 20 January 2021

Citation:

Luo J, Li L, Wang T, Yang K, Feng Y, Yang $R, M a Y$, Gao $P$, Yang $B$ and Jiao L (2021) Risk Factors of New

Cerebral Infarctions After Endovascular Treatment for Basilar

Artery Stenosis Based on

High-Resolution Magnetic Resonance

Imaging. Front. Neurol. 11:620031.

doi: 10.3389/fneur.2020.620031
Objective: The current study aims to analyze the risk factors of new cerebral infarctions in the distribution of basilar artery (BA) detected by diffusion-weighted imaging (DWI) after endovascular treatment in patients with severe BA stenosis.

Methods: Data was collected from the electronic medical records of patients with severely atherosclerotic basilar artery stenosis $(\geq 70 \%)$ who underwent endovascular treatment. The plaque characteristics, including the plaque distribution, plaque burden, plaque enhancement index, remodeling ratio, and stenosis degree, were evaluated qualitatively and quantitatively using high-resolution magnetic resonance imaging (HR-MRI) and digital subtraction angiography (DSA). The characteristics of the procedure, such as the type of treatment, balloon diameter, balloon length, stent diameter, and stent length, were analyzed.

Results: A total of 107 patients with severe basilar artery stenosis $(\geq 70 \%)$ who underwent endovascular treatment were enrolled. The study participants included 77 men and 30 women, with an average age of $61.6 \pm 8.1$ years. The rate of postoperative new cerebral infarctions was 55.1\% (59/107), of which $74.6 \%$ (44/59) were caused by artery-to-artery embolism, 6.8\% (4/59) due to perforator occlusion, and 18.6\% (11/59) were caused by a mixed mechanism. Twelve of 59 patients had ischemic events, with nine cases of stroke and three cases of transient ischemic attacks (TIA). The plaque burden in the DWI-positive group was significantly larger than that in the DWI-negative group $(3.7 \%$ vs. $-8.5 \%, p=0.016)$. Positive remodeling was more common in the DWI-positive group than in the DWI-negative group (35.6\% vs. $16.7 \%, p=0.028)$. Smoking was inversely correlated with the rate of new cerebral infarctions (odds ratio, 0.394; 95\% confidence interval, 0.167-0.926; $p=0.033$ ).

Conclusion: The plaque characteristics are not associated with new cerebral infarctions in the distribution of BA, although a large plaque burden and positive remodeling are more 
likely to appear in patients with new cerebral infarctions after BA stenting, which warrants further studies with a larger sample size. As for smoking, the inverse correlation with new cerebral infarctions in the BA territory needs large-scale prospective randomized controlled trials to verify.

Keywords: basilar artery stenosis, new cerebral infarctions, high-resolution magnetic resonance imaging, smoking, plaque burden, remodeling index

\section{INTRODUCTION}

Basilar artery (BA) atherosclerotic stenosis is a common cause of transient ischemic attacks (TIA) and stroke, accounting for approximately $20 \%$ of symptomatic ischemic infarctions of the posterior circulation (1). Endovascular therapy is an effective alternative treatment for drug-refractory BA stenosis. However, endovascular treatment for BA atherosclerotic stenosis has a high perioperative complication rate. The Stenting and Aggressive Medical Management for Preventing Recurrent Stroke in Intracranial Stenosis (SAMMPRIS) trial described that the stroke or death rate due to $\mathrm{BA}$ atherosclerotic stenosis was as high as $21.6 \%$ after BA stenting (2). However, the new cerebral infarction rate detected by diffusion-weighted imaging (DWI) may be higher given that the definition of stroke is the combination of new cerebral infarctions on DWI and clinical symptoms of neurological deficits, as well as some new cerebral infarctions that have no clinical symptoms and are only detected by DWI (3). Indeed, some studies have reported that the prevalence of new cerebral infarctions discovered by DWI is as high as $70 \%$ after endovascular treatment $(4,5)$.

Although most new cerebral infarctions after endovascular treatment are silent without clinical symptoms, the long-term risk of neurological deficits is enormous. Several studies have found that silent infarctions can lead to subcortical cavities, cortical atrophy, and glial cell proliferation, resulting in longterm complications such as cognitive decline, impaired motor coordination, poor visual reactivity, mental disorders, and even a high risk of long-term stroke $(6,7)$. Indeed, previous studies have reported that silent infarctions increase the risk of recurrent stroke by 2-3-fold, despite strict control of vascular risk factors (8). Therefore, it is crucial to identify the risk of new cerebral infarctions after endovascular treatment.

Previous studies have shown that silent cerebral infarctions are associated with advanced age, hypertension, and diabetes mellitus in the natural course $(7,9)$. However, few studies have focused on exploring the risks of new cerebral infarctions in the BA territory after endovascular treatment for BA stenosis, in particular, the relationship between plaque characteristics and new cerebral infarctions. High-resolution magnetic resonance imaging (HR-MRI) is an imaging evaluation technology for plaque characteristics that has emerged in recent years. HR-MRI has advantages including high resolution, visualization of the vascular wall structure, and non-invasiveness, and is considered a reliable evaluation method for evaluating plaque characteristics (10). Furthermore, HR-MRI can be used to assess the plaque location, burden, enhancement ratio, size, length, and area, as well as the remodeling index both qualitatively and quantitatively
(11-13). In the current study, we retrospectively explored the risk factors of new cerebral infarctions in the BA territory detected by DWI after endovascular treatment for BA stenosis based on HR-MRI in Chinese individuals. We also sought to determine the risk factors associated with patient demographics and the endovascular treatment procedure.

\section{MATERIALS AND METHODS}

This study was conducted in accordance with the Good Clinical Practice guidelines and the ethical principles of the Declaration of Helsinki. The study was approved by the Ethics Committee of our center, and given the retrospective nature of the study and the fact that data were analyzed anonymously, the study was exempted from obtaining consent from patients. The demographic characteristics, plaque imaging features, and procedure characteristics were examined to analyze the risk of new cerebral infarctions detected by DWI.

\section{Study Populations and Demographics}

We retrospectively enrolled patients with basilar atherosclerotic stenosis who were treated by endovascular therapy between January 1, 2012, and December 31, 2019. The inclusion criteria were as follows: (1) Patients with symptomatic atherosclerotic stenosis of the BA aged from 18 to 80 years, (2) the degree of stenosis of the lesions was more than $70 \%$ confirmed by digital subtraction angiography (DSA), (3) HR-MRI was performed before intervention, and (4) the sequence of DWI was performed $72 \mathrm{~h}$ before and after the surgery. Some patients were excluded based on the following criteria: (1) Acute BA occlusion treated by endovascular therapy; (2) endovascular therapy for another intracranial and extracranial vessel disease simultaneously; (3) BA stenosis accompanied with moderate-tosevere stenosis of the vertebral artery; (4) non-atherosclerotic cause of BA stenosis, such as Moyamoya disease, vasculitis, or vascular dissection; and (5) preoperative DWI suggested largearea cerebral infarctions $(\geq 1 / 2)$.

The demographic data of patients were collected, including age, gender, body mass index (BMI), vascular risk factors (hypertension, diabetes, hyperlipidemia, coronary heart disease [CHD], qualifying events, smoking history, and drinking history), modified Rankin score (mRS) at admission and discharge, and preoperative and postoperative results of DWI. The evaluation of the new infarction was based on a new high signal on DWI and a new low signal on apparent diffusion coefficient (ADC) imaging after $72 \mathrm{~h}$ of intervention compared with pre-operative imaging. If a new infarction was 
detected in the blood supply area of the BA, such as the brainstem, cerebellum, occipital lobe, or thalamus, the patient was classified as DWI positive (DWI+). If the patient had new infarctions in other districts or no new infarction, the patient was classified as DWI negative (DWI-). As for smoking history, nonsmokers were defined as not smoking currently or previously, or as smokers.

\section{Imaging Protocol and Analysis}

All eligible patients were examined by a $1.5 \mathrm{~T}$ or $3.0 \mathrm{~T}$ magnetic resonance imaging (MRI) system (MAGNETOM Avanto; Siemens Healthineers) with a standard 8-channel head coil to assess the characteristics of BA stenosis. Multi-sequence scans were performed as follows: time-of-flight magnetic resonance angiography (TOF-MRA), fast spin-echo T1-weighted imaging (T1WI-FSE), and T1-weighted enhanced imaging (T1WI + C). The coronal acquisition parameters were as follows: repetition time, $550 \mathrm{~ms}$; echo time, $27 \mathrm{~ms}$; field of view, $200 \times 200 \mathrm{~cm}$; layer thickness, $0.6 \mathrm{~mm}$, and echo-train length, 158. The T1WI + C sequence was scanned 5 min after the intravenous injection of gadopentetate meglumine. All images required reconstruction for image analysis from axial, coronal, and sagittal views.

All plaque images were analyzed using CMRtools (Cardiovascular Imaging Solutions Ltd., UK) by two experienced neuroradiologists who were not involved in statistical analyses. The original data with the type of digital imaging and communications in medicine (DICOM) were required for image analysis. The measurements of the vessel area (VA) and lumen area (LA) were performed on cross-sectional T1-weighted BA images at the maximal lumen narrowing (MLN) or reference sites after zooming in 400 times. We used two lines to divide the axial lumen into four quadrants: ventral, dorsal, left, and right $(11,13)$. The plaque was considered to belong in the quadrant which had the thickest part of the plaque. If the plaque had a large span and the thickest part was between two quadrants, it was defined as being distributed in more than two quadrants. The reference site was selected at the normal segment of proximal or distal stenosis according to the criteria of WASID (Warfarin vs. Aspirin for Symptomatic Intracranial Disease) where the proximal segment was preferred, but the distal vessel was considered when the proximal segment was diseased (14). Wall area (WA) was measured using VA-LA, and the plaque burden was defined as, [(WA $\mathrm{MLN}_{-}-\mathrm{WA}$ reference $) / \mathrm{VA}$ MLN $] \times 100 \%$. The remodeling index (RI) was defined as, [VA MLN/VA reference]; RI $\geq 1.05$ was defined as positive remodeling, $\mathrm{RI} \leq 0.95$ as negative remodeling, and RI between 0.95 and 1.05 as intermediate remodeling. The contrast enhancement ratio was measured at the slice of the MLN that was normalized by the signal from adjacent gray matter. The enhancement ratio was calculated by, [signal of plaque (postcontrast)/signal of gray matter (post-contrast)]/[signal of plaque (pre-contrast)/signal of gray matter (pre-contrast)] $\times 100 \%(15)$.

\section{Interventional Procedure and Analysis}

The culprit lesions were treated by neurosurgeons who had more than 15 years of experience with endovascular treatment, including primary angioplasty, balloon-mounted stent placement (Apollo), and self-expansion stent placement (Gateway-Wingspan system). The therapeutic strategies were decided by the operators according to the characteristics of the lesion and their own experience; more details are provided in a previous study (16). All patients received preoperative medication with a combination of aspirin ( $100 \mathrm{mg}$ daily) and clopidogrel ( $75 \mathrm{mg}$ daily) started $5 \mathrm{~d}$ prior to the procedure, or a loading dose of aspirin and clopidogrel (300 mg each) $1 \mathrm{~d}$ before the procedure. During the procedure, systematic heparin was administered by intravenous injection at a dose of $2 / 3 \mathrm{mg}$ per $\mathrm{kg}$ of body weight. The standard of procedure was referred to as the protocol of CASSISS (China Angioplasty and Stenting for Symptomatic Intracranial Severe Stenosis) (17). The three types of intervention, primary angioplasty, self-expansion stent (SES), and balloon-mounted stent (BMS), had different procedures. Angiography with a high-pressure contrast agent was followed by balloon pre-expansion during primary angioplasty. As for SES placement, the procedure was similar to that for primary angioplasty except for the self-expansion stent placement after angiography. As for BMS placement, the balloon expanded the stent mostly without the need for pre-expansion.

The parameters of the procedure were collected, including the vessel diameter (VD) at the most stenotic site and reference site, length of the lesion, intervention method, diameter and length of the balloon and/or stent, and pressure $(\mathrm{P})$ before and/or after expansion. The degree of stenosis was defined as [ 1 $\mathrm{VD}$ MLN $\left./ \mathrm{VD}_{\text {reference }}\right) \times 100 \%$ ] in accordance with the standard of WASID (14). We also defined new composite variables to explore the relationship between the lesion and instruments. The diameter ratio was defined as the maximal diameter of the implant divided by the VD $\mathrm{MLN}_{\mathrm{N}}$ and VD reference, respectively.

The BA was divided into three segments according to the branch of the anterior inferior cerebellar artery and the superior cerebellar artery: low, middle, and high segments (18).

\section{Statistical Analysis}

Statistical analyses were performed using SPSS 25.0 (IBM). Continuous variables are described as means \pm SD and categorical variables as frequency and percentage. Student's $t$ test or Mann-Whitney $U$ test was used for the comparison of continuous variables, and the chi-square test was used for categorical variables. Covariates with a univariate $p$-value $<0.10$ were subsequently enrolled in multivariate logistic regression analysis. The odds ratio (OR) with $95 \%$ confidence interval (CI) was determined using a logistic regression model. $P$-values $<0.05$ were considered to indicate statistical significance.

\section{RESULTS}

\section{Demographic Characteristics}

A total of 376 consecutive patients with BA stenosis underwent endovascular treatment at our center from January 1, 2012, to December 31, 2019; among them, 107 patients were eligible after screening (see Figure 1). The patients comprised 77 men and 30 women with an average age of $61.6 \pm 8.1$ years. The success rate of endovascular therapy was $100 \%$. The $\mathrm{mRs}$ of most patients ranged from 0 to 1 , with a proportion 


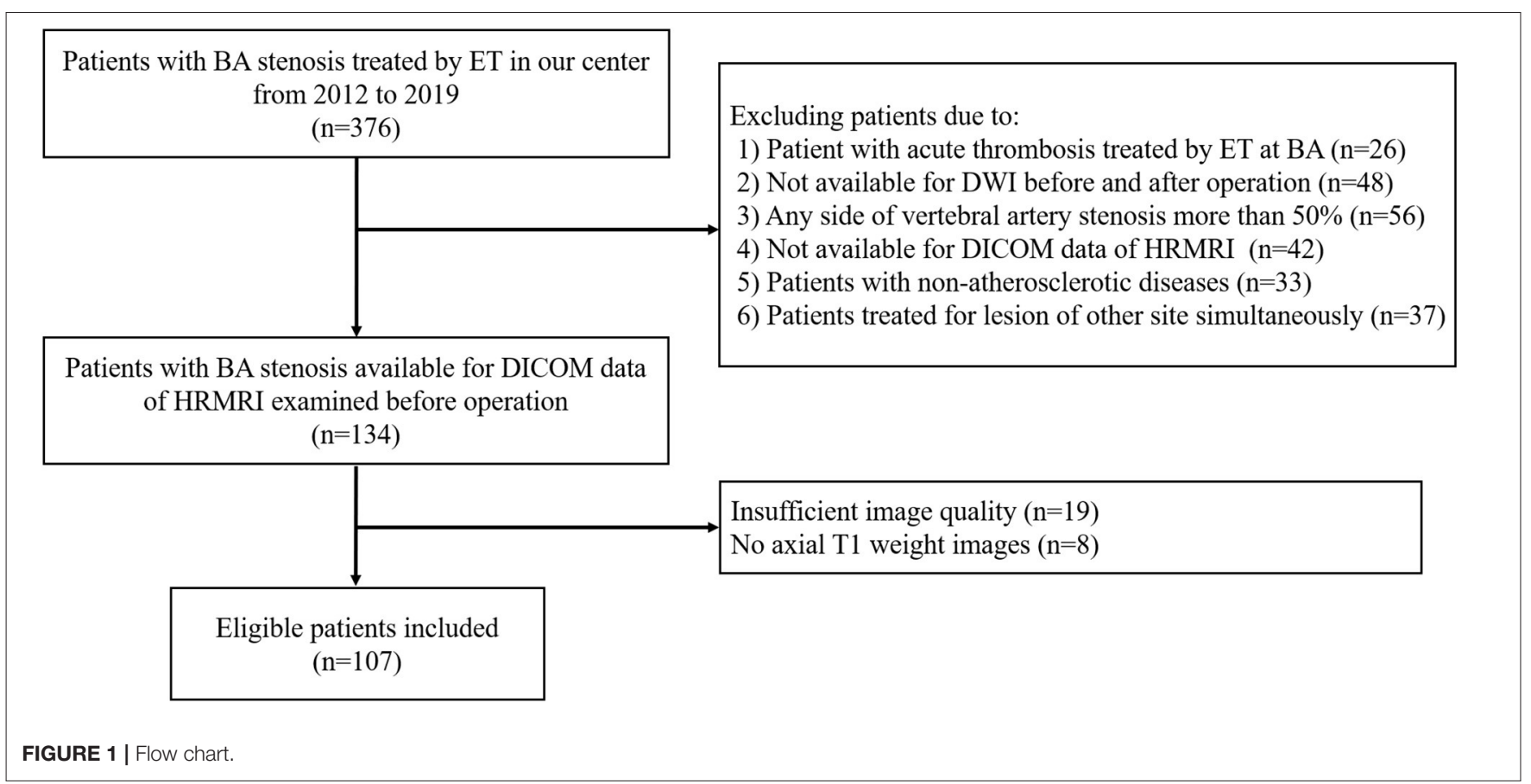

of $86.3 \%$. Fifty-nine patients (55.1\%) developed new cerebral infarctions after endovascular therapy as evaluated by DWI; among these, 12 patients had ischemic events, with nine cases of stroke and three cases of TIA. There was no death at discharge. As for the mechanisms of infarctions, $74.6 \%$ of infarctions (44/59) were caused by artery-to-artery embolism, $6.8 \%(4 / 59)$ by perforator occlusion, and $18.6 \%(11 / 59)$ by a mixed mechanism. There was a significant difference in smoking history $(p=0.021)$ between DWI- and DWI+ patients. The detailed demographic characteristics are described in Table 1.

\section{Characteristics Based on HRMRI, DSA, and the Procedure of Endovascular Treatment}

The average stenosis of patients was $77.8 \pm 6.8 \%$, with $57.9 \%$ of lesions presented at the middle segment of BA from the coronal view and $34.6 \%$ at the ventral BA from the axial view. Half of the lesions were eccentric, while the rest presented as either $\mathrm{C}$ type or circular type. Most lesions had nonpositive remodeling, with a proportion of $72.9 \%$. More than half of the BA stenosis (57.9\%) was treated with self-expansion stenosis. The most common site of the lesions was the middle segment of the BA (57.9\%) and the ventral region of the BA (34.6\%) (Figures 2, 3). There were significant differences in wall area of the reference vessel $\left(17.1 \mathrm{~mm}^{2}\right.$ vs. $15.1 \mathrm{~mm}^{2}, p$ $=0.040)$, plaque burden $(-8.5 \%$ vs. $3.7 \%, p=0.016)$, and positive remodeling $(16.7 \%$ vs. $35.6 \%, p=0.028)$ between DWI- and DWI+ patients. More details are presented in Tables 2, 3.

\section{Multivariate Analysis}

According to the results of conventional statistical analysis with $p<0.10$, these covariates, including CHD, smoking history,
TABLE 1 | Demographic Characteristics.

\begin{tabular}{lcccc}
\hline Variables & $\begin{array}{c}\text { All patients } \\
(\boldsymbol{n}=\mathbf{1 0 7})\end{array}$ & $\begin{array}{c}\text { DWI- } \\
(\boldsymbol{n}=\mathbf{4 8})\end{array}$ & $\begin{array}{c}\text { DWI+ } \\
(\boldsymbol{n}=\mathbf{5 9})\end{array}$ & $\boldsymbol{P}$-value \\
\hline Age (y) & $61.6 \pm 8.1$ & $60.7 \pm 8.3$ & $62.4 \pm 7.9$ & 0.296 \\
Male & $77(72.0 \%)$ & $37(77.1 \%)$ & $40(67.8 \%)$ & 0.287 \\
BMl & $26.4 \pm 3.0$ & $26.3 \pm 3.0$ & $26.4 \pm 3.0$ & 0.875 \\
Hypertension & $88(82.2 \%)$ & $39(81.3 \%)$ & $49(83.1 \%)$ & 0.808 \\
Diabetes Mellitus & $46(43.0 \%)$ & $18(37.5 \%)$ & $28(47.5 \%)$ & 0.301 \\
Hyperlipidemia & $19(17.8 \%)$ & $9(18.8 \%)$ & $10(16.9 \%)$ & 0.808 \\
CHD & $13(12.1 \%)$ & $3(6.3 \%)$ & $10(16.9 \%)$ & 0.092 \\
Arrhythmia & $4(3.7 \%)$ & $2(4.2 \%)$ & $2(3.4 \%)$ & 0.609 \\
Smoking history & $47(43.9 \%)$ & $27(56.3 \%)$ & $20(33.9 \%)$ & 0.021 \\
Drinking history & $29(27.1 \%)$ & $13(27.1 \%)$ & $16(27.1 \%)$ & 0.997 \\
Qualifying event & & & & 0.487 \\
$\quad$ TIA & $13(12.1 \%)$ & $7(14.6 \%)$ & $6(10.2 \%)$ & \\
$\quad$ Stroke & $94(87.9 \%)$ & $41(85.4 \%)$ & $53(89.8 \%)$ & \\
Pre-operation mRs & & & & 0.059 \\
$\quad<2$ & $93(86.9 \%)$ & $45(93.8 \%)$ & $48(81.4 \%)$ & \\
$\quad 22$ & $14(13.1 \%)$ & $3(6.2 \%)$ & $11(18.6 \%)$ & \\
\hline
\end{tabular}

BMI, body mass index; CHD, coronary heart disease; mRS, modified Rankin Scale.

preoperative mRs, plaque burden, and remodeling index, were included in the multivariate logistic regression analysis. Although the $p$-values of the vessel area and wall area at the reference site were $<0.10$, they were not included in the multivariate analysis because the plaque burden and remodeling type were calculated. Multivariate analysis demonstrated that smoking was an independent factor for new cerebral infarctions (OR, 0.394; 95\% CI, 0.167-0.926, $p=0.033$ ). More details are presented in Table 4. 


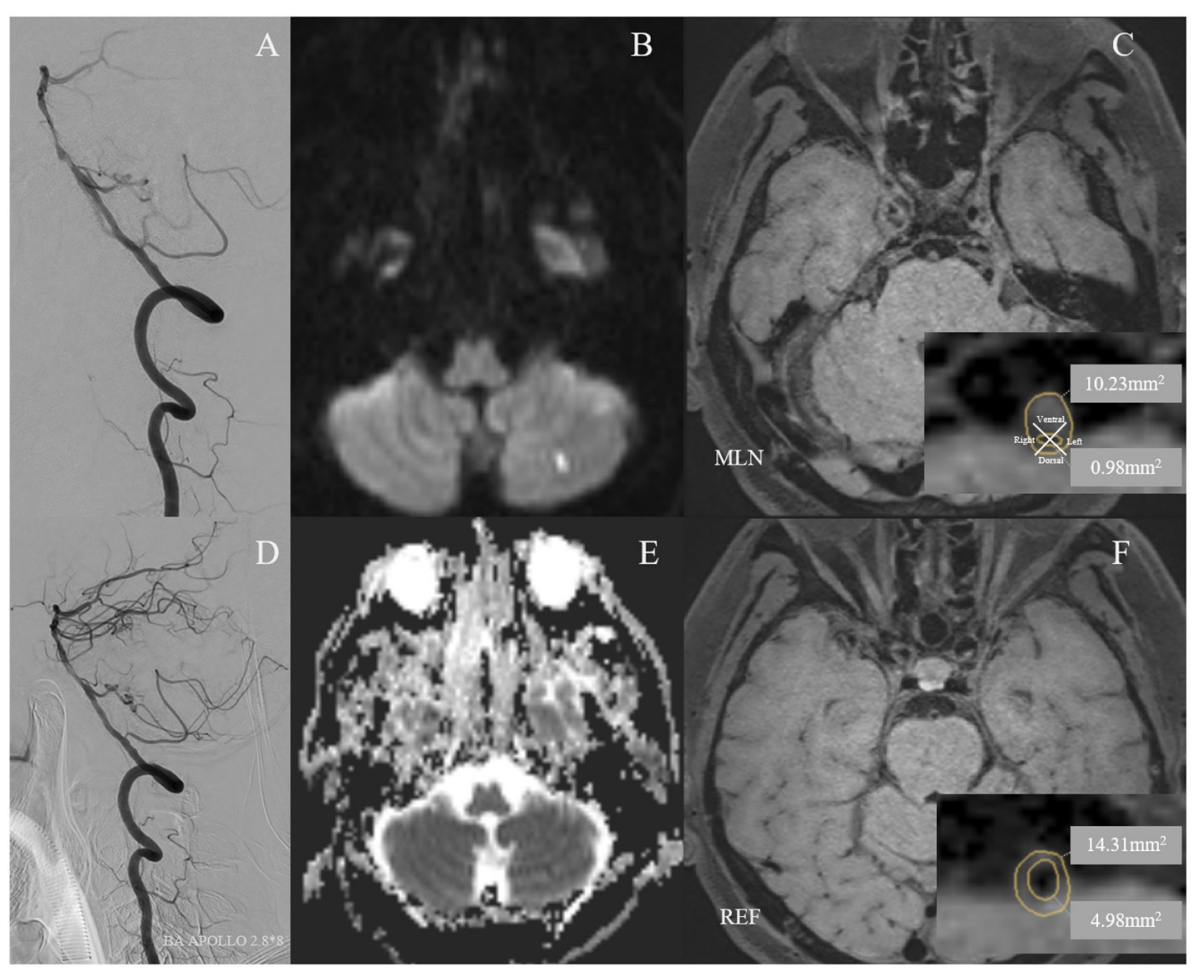

FIGURE 2 | A 59-year-old man with cigarette smoking history and drinking history presented with recurrent transient attacks of dizziness for 2 months. (A) Pre-operative digital subtraction angiography (DSA) showed stenosis at the middle segment of the basilar artery (BA) with $75.5 \%$ of degree. (D) A $2.8 \times 8$ mm Apollo balloon-mounted stent (MicroPort NeuroTech, Shanghai, China) was placed at the stenotic segment. The patient had a new cerebral infarction at the left cerebellum within $72 \mathrm{~h}$ after stenting without clinical symptoms, which was detected by diffusion-weighted imaging with a new high signal (B) and apparent diffusion coefficient imaging with a new low signal (E). Figures of the right column are cross-sectional T1-weighted BA images at the maximal lumen narrowing (MLN) (C) and reference (REF) (F) sites. The plaque was eccentric and belonged to the ventral side of the BA (C). Vessel area (VA) and lumen area (LA) at the MLN (C), VA 10.23 mm², LA 0.98 $\mathrm{mm}^{2}$ ) or REF (F, VA $14.31 \mathrm{~mm}^{2}$, LA $4.98 \mathrm{~mm}^{2}$ ) sites were manually traced for measuring after zooming in 400 times. Wall area (WA) at the MLN or REF sites was calculated by VA-LA. The plaque burden was calculated as [(WA MLN-WA REF $)$ NA MLN] $_{\text {M }} \times 100 \%$ and the remodeling index was calculated as VA MLN $_{\text {NA }}$ REF. Therefore, the plaque burden was $-0.78 \%$. The remodeling index of the vessel at MLN was 0.71 , which was categorized as negative remodeling.

\section{DISCUSSION}

The impact of new cerebral infarctions has been largely ignored in clinical practice, especially those cases without clinical symptoms. Most clinical studies, including multicenter randomized controlled studies, only regarded symptomatic cerebral infarctions (stroke) or TIA as the endpoint, which reduces the public's knowledge of new cerebral infarctions without clinical symptoms, known as silent cerebral infarctions $(19,20)$. Although some new cerebral infarctions have no symptoms, they still pose as a high-risk factor for stroke in the future (8). In addition, silent cerebral infarctions can cause longterm cognitive impairment, impaired motor coordination, and mental illness $(6,21,22)$. Therefore, some experts have suggested that the definition of silent cerebral infarctions should be replaced by covert cerebral infarctions, which are difficult to detect by contemporary evaluation methods (23).

The history of endovascular treatment may rapidly increase the probability of new cerebral infarctions without clinical symptoms. However, few studies have reported the incidence of new cerebral infarction after endovascular treatment for BA stenosis. In the current study, the incidence of new cerebral infarctions in the distribution of BA was $55.1 \%$, with $43.9 \%$ of them being silent cerebral infarctions, which is consistent with the rate of $15-70 \%$ of new cerebral infarctions after carotid stenting $(5,24)$. Artery-to-artery embolism was the most common infarction mechanism, similar to the findings of previous studies (25). The results demonstrated that smoking, plaque burden, and positive remodeling may be the influencing factors for new cerebral infarctions. Smoking was independently associated with new cerebral infarctions.

\section{Smoking Is Inversely Associated With the Risk of New Cerebral Infarctions After Endovascular Treatment}

As we all know, smoking is a classic risk factor for cardiovascular diseases. Indeed, some studies have reported that the 10year risk of death was 2 -fold in smokers compared to that in non-smokers; smoking has been shown to be the most important risk factor for premature death $(26,27)$. The damaging effect of smoking has been attributed to several dysfunctions, including endothelial dysfunction, inflammation, and a state of prothrombotic formation $(28,29)$. 


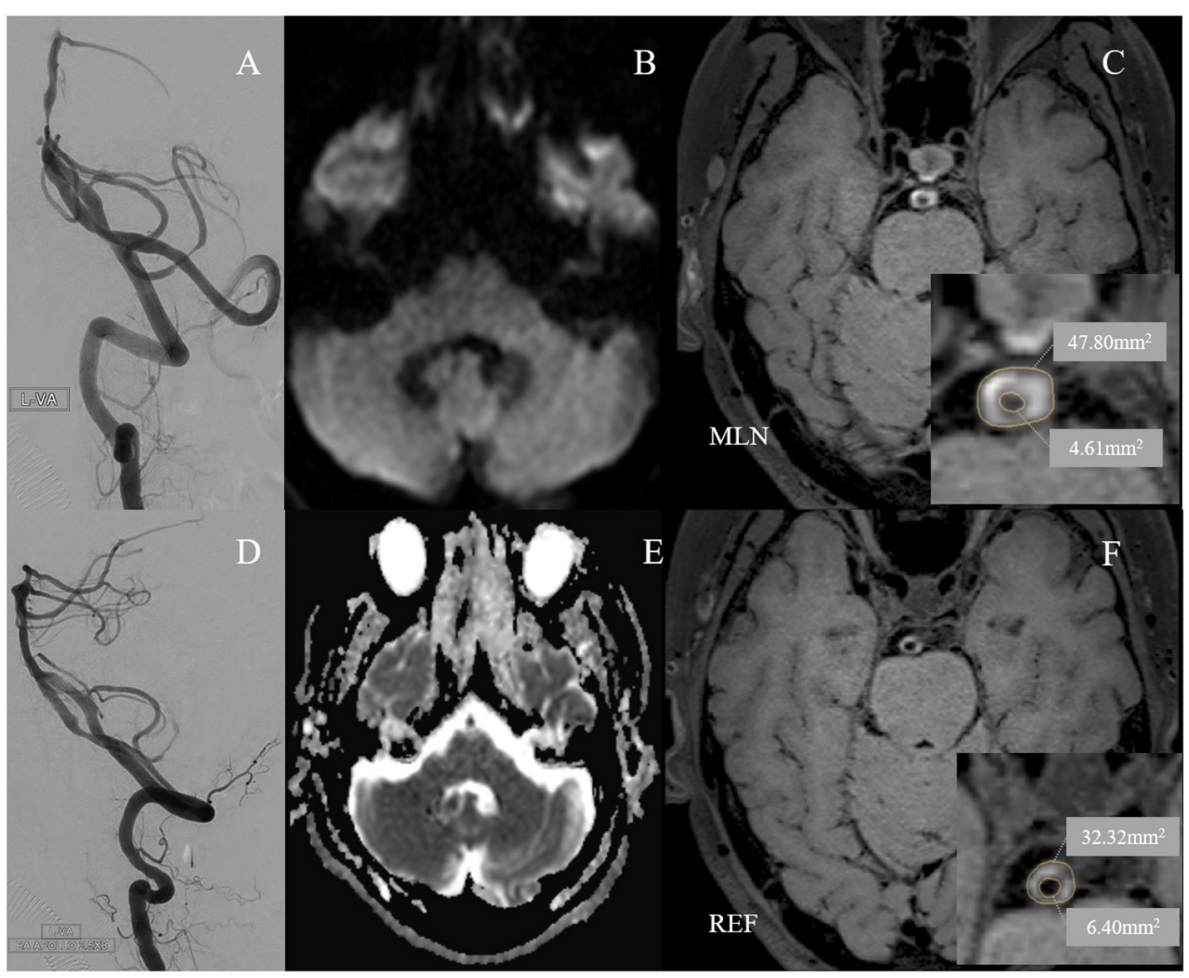

FIGURE 3 | A 60-year-old man with cigarette smoking history presented with recurrent transient attacks of vertigo for 1 month. (A) Pre-operative digital subtraction angiography (DSA) showed stenosis at the low segment of the basilar artery (BA) with $77.7 \%$ of degree. (D) A $2.5 \times 8$ mm Apollo balloon-mounted stent (MicroPort NeuroTech, Shanghai, China) was placed at the stenotic segment. There was no new cerebral infarction confirmed by diffusion-weighted imaging (B) and apparent diffusion coefficient imaging (E) within $72 \mathrm{~h}$ after stenting. Figures of the right column are cross-sectional T1-weighted BA images at the maximal lumen narrowing (MLN) (C) and reference (REF) (F) sites. The plaque was concentric and belonged to the circular type (C). Vessel area (VA) and lumen area (LA) at the MLN (CC, VA $47.80 \mathrm{~mm}^{2}$, LA $4.61 \mathrm{~mm}^{2}$ ) or REF (F, VA $32.32 \mathrm{~mm}^{2}$, LA $6.40 \mathrm{~mm}^{2}$ ) sites were manually traced for measuring after zooming in 400 times. Wall area (WA) at the MLN or REF sites was calculated by VA-LA. The plaque burden was calculated as [(WA $\left.\left.{ }_{M L N}-W A_{R E F}\right) / V A_{M L N}\right] \times 100 \%$ and the remodeling index was calculated as VA MLN NA REF. Therefore, the plaque burden was $36.1 \%$. The remodeling index of the vessel at MLN was 1.48, which was categorized as positive remodeling.

Unexpectedly, there was a paradoxical phenomenon similar to our finding in a large number of studies. Some studies on fibrinolytic therapy for cardiovascular diseases have shown that smokers are associated with better in-hospital and short-term follow-up outcomes. The results of the multivariate analysis demonstrated that smoking was associated with better short-term outcomes (adjusted OR, 0.80; 95\% CI, 0.72-0.90) (30). One of the hypotheses was that thrombosis formation was more common in smokers. Another interpretation was that smokers with acute stroke were younger than non-smokers and had lower rates of complications, such as diabetes mellitus and hypertension. Similarly, in the era of endovascular treatment for cardiovascular diseases, the paradoxical effect of smoking was demonstrated in landmark clinical trials, where the short-term prognosis for smokers with acute cardiovascular diseases after mechanical thrombectomy was better than that for non-smokers (adjusted OR: 0.54, 95\% CI: 0.38-0.76) (31, 32). Furthermore, previous studies of a subgroup analysis of the International Carotid Stenting Study (ICSS) demonstrated a reduced incidence of 30day postoperative major adverse events in smokers compared with non-smokers (adjusted OR, 0.33; 95\% CI, 0.13-0.85) (33). Some studies have given some explanations for this phenomenon.
Dual antiplatelet therapy, including aspirin and clopidogrel, is an essential preoperative preparation for endovascular treatment with conventional or loading doses. Tobacco smoking could enhance the antiplatelet effects of clopidogrel by affecting the enzyme responsible for converting clopidogrel into its active form, which increases the ability to resist platelet aggregation and reduces the probability of thrombogenesis $(34,35)$. Meanwhile, some researchers suspected that smoking may create a vascular disease state that is more responsive to clopidogrel, even when the patients have not smoked for several years (36).

\section{Plaque Characteristics Associated With New Cerebral Infarctions After Endovascular Treatment}

Although plaque characteristics were insignificant in the multivariate analysis, we found that large plaque burden and positive remodeling was higher in the DWI+ group than in the DWI- group in accordance with univariate analysis. The greater the plaque burden with a large lipid core, the higher the risk of new cerebral infarctions after endovascular treatment, because the risk of the plaque breaking increases during 
TABLE 2 | Lesion characteristics based on HRMRI and DSA.

\begin{tabular}{|c|c|c|c|c|}
\hline Variables & $\begin{array}{l}\text { All patients } \\
(n=107)\end{array}$ & $\begin{array}{c}\text { DWI- } \\
(n=48)\end{array}$ & $\begin{array}{c}\text { DWI+ } \\
(n=59)\end{array}$ & $P$-value \\
\hline Stenosis site (coronal view) & & & & 0.506 \\
\hline Low & 40 (37.4\%) & $19(39.6 \%)$ & $21(35.6 \%)$ & \\
\hline Middle & 62 (57.9\%) & 28 (58.3\%) & $34(57.6 \%)$ & \\
\hline Low \& Middle & $5(4.7 \%)$ & $1(2.1 \%)$ & 4 (6.8\%) & \\
\hline Stenosis site (axial view) & & & & 0.141 \\
\hline Ventral & 37 (34.6\%) & 11 (22.9\%) & 26 (44.1\%) & \\
\hline Side & $31(29.0 \%)$ & $16(33.3 \%)$ & 15 (25.4\%) & \\
\hline Dorsal & $18(16.8 \%)$ & $9(18.8 \%)$ & $9(15.5 \%)$ & \\
\hline$\geq 2$ quadrant & $21(19.6 \%)$ & $12(25.0 \%)$ & $9(15.3 \%)$ & \\
\hline Plaque morphology & & & & 0.245 \\
\hline Eccentric & 58 (54.2\%) & $29(60.4 \%)$ & 29 (49.2\%) & \\
\hline C type and circular & $49(45.8 \%)$ & $19(39.6 \%)$ & $30(50.8 \%)$ & \\
\hline Tandem lesion & 8 (7.5\%) & $2(4.2 \%)$ & $6(10.2 \%)$ & 0.240 \\
\hline Stenosis (\%) & $77.8 \pm 6.8 \%$ & $77.8 \pm 6.2 \%$ & $77.8 \pm 7.5 \%$ & 0.961 \\
\hline Diameter at MLN (mm) & $0.6 \pm 0.2$ & $0.6 \pm 0.2$ & $0.6 \pm 0.2$ & 0.723 \\
\hline Diameter at reference $(\mathrm{mm})$ & $2.8 \pm 0.6$ & $2.7 \pm 0.6$ & $2.7 \pm 0.5$ & 0.565 \\
\hline Plaque length (mm) & $6.6 \pm 3.2$ & $6.2 \pm 2.8$ & $6.9 \pm 3.5$ & 0.369 \\
\hline $\begin{array}{l}\text { Plaque length/Degree of } \\
\text { stenosis }\end{array}$ & $8.5 \pm 3.8$ & $8.0 \pm 3.5$ & $8.9 \pm 4.1$ & 0.273 \\
\hline VA at $\mathrm{MLN}\left(\mathrm{mm}^{2}\right)$ & $18.6 \pm 7.6$ & $18.4 \pm 7.9$ & $18.5 \pm 7.1$ & 0.666 \\
\hline LA at MLN $\left(\mathrm{mm}^{2}\right)$ & $1.2 \pm 0.7$ & $1.2 \pm 0.8$ & $1.2 \pm 0.7$ & 0.757 \\
\hline WA at MLN $\left(\mathrm{mm}^{2}\right)$ & $17.4 \pm 7.4$ & $17.2 \pm 7.8$ & $17.3 \pm 6.8$ & 0.539 \\
\hline VA at reference $\left(\mathrm{mm}^{2}\right)$ & $21.9 \pm 7.0$ & $23.2 \pm 7.3$ & $20.8 \pm 6.7$ & 0.076 \\
\hline$L A$ at reference $\left(\mathrm{mm}^{2}\right)$ & $5.9 \pm 2.6$ & $6.1 \pm 2.6$ & $5.7 \pm 2.6$ & 0.324 \\
\hline WA at reference $\left(\mathrm{mm}^{2}\right)$ & $16.0 \pm 5.0$ & $17.1 \pm 5.4$ & $15.1 \pm 4.5$ & 0.040 \\
\hline Plaque burden (\%) & $-1.8 \pm 40.1$ & $-8.5 \pm 33.2$ & $3.7 \pm 44.4$ & 0.016 \\
\hline Remodeling type & & & & 0.028 \\
\hline Positive remodeling & $29(27.1 \%)$ & $8(16.7 \%)$ & $21(35.6 \%)$ & \\
\hline Non-positive remodeling & $78(72.9 \%)$ & 40 (83.3\%) & 38 (64.4\%) & \\
\hline Enhancement ratio & $1.7 \pm 0.6$ & $1.7 \pm 0.6$ & $1.8 \pm 0.7$ & 0.965 \\
\hline
\end{tabular}

MLN, maximal lumen narrowing; VA, vessel area; LA, lumen area; WA, wall area.

endovascular treatment with external force (37). Similarly, the remodeling index was associated with the risk of new cerebral infarctions. Most studies have defined a remodeling index $>1.05$ as positive remodeling with plaque advance outward, and a remodeling index $<0.95$ as negative remodeling with plaque advance inward. In the current study, more than half of patients (65.4\%) were negative remodeling, followed by positive remodeling $(27.1 \%)$ and intermediate remodeling $(8.4 \%)$. The plaque burden in positive remodeling was larger than that in negative remodeling. Therefore, the risk of new cerebral infarctions was higher in patients with positive remodeling. Some studies have demonstrated that plaque stability is associated with a small plaque burden and negative remodeling. The unstable plaque increased the risk of plaque breakage during endovascular treatment and the rate of new postoperative infarctions (3840). Furthermore, previous studies have suggested that positive remodeling is more common in patients with advanced BA stenosis (38). However, we found that negative remodeling was a common condition in patients with symptomatic BA stenosis
TABLE 3 | Characteristics of procedure.

\begin{tabular}{|c|c|c|c|c|}
\hline Variables & $\begin{array}{c}\text { Total } \\
(n=107)\end{array}$ & $\begin{array}{c}\text { DWI- } \\
(n=48)\end{array}$ & $\begin{array}{c}\text { DWI+ } \\
(n=59)\end{array}$ & $P$-value \\
\hline \multicolumn{5}{|l|}{ Treatment type } \\
\hline PA & $21(19.6 \%)$ & $10(20.8 \%)$ & $11(18.6 \%)$ & 0.943 \\
\hline BMS & 24 (22.4\%) & $11(22.9 \%)$ & 13 (22.0\%) & \\
\hline SES & $62(57.9 \%)$ & $27(56.3 \%)$ & $35(59.3 \%)$ & \\
\hline Diameter max of PTAS (mm) & $3.1 \pm 0.8$ & $3.0 \pm 0.8$ & $3.1 \pm 0.8$ & 0.641 \\
\hline 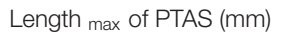 & $13.6 \pm 4.2$ & $13.5 \pm 4.3$ & $13.7 \pm 4.2$ & 0.832 \\
\hline Pressure $\max$ of PTAS (atm) & $6.4 \pm 1.5$ & $6.4 \pm 1.4$ & $6.4 \pm 1.6$ & 0.869 \\
\hline Diameter ratio of stenosis* & $5.8 \pm 2.9$ & $5.5 \pm 2.5$ & $6.0 \pm 3.2$ & 0.446 \\
\hline Diameter ratio of reference ${ }^{*}$ & $1.2 \pm 0.3$ & $1.0 \pm 0.3$ & $1.2 \pm 0.3$ & 0.441 \\
\hline
\end{tabular}

PA, primary angioplasty; BMS, balloon-mounted stent; SES, self-expansion stent; PTAS, percutaneous trans/uminal angioplasty with or without stenting.

*The diameter ratio was defined as the maximal diameter of implant divided by vessel diameter of stenosis and reference.

TABLE 4 | Risks for post-operative new cerebral infarction assessed by multivariate analysis.

\begin{tabular}{lcc}
\hline & OR (95\% Cl) & $P$-value \\
\hline Age & $1.003(0.952-1.057)$ & 0.905 \\
Smoking history & $0.394(0.167-0.926)$ & 0.033 \\
CHD & $4.109(0.973-17.354)$ & 0.055 \\
Pre-operative mRs & - & \\
$\quad<2$ & $2.710(0.66-11.120)$ & 0.166 \\
$\geq 2$ & $1.001(0.988-1.014)$ & 0.988 \\
Plaque burden & - & - \\
Remodeling ratio & - & - \\
$\quad$ Non-positive remodeling & $2.807(0.832-9.472)$ & 0.096 \\
\hline
\end{tabular}

CHD, coronary heart disease.

treated by endovascular therapy. The reason for the difference in results was the diversity of eligible patients enrolled in the study. Non-acute, stable patients with symptomatic severe BA stenosis were admitted to our center for endovascular treatment. Studies have found that the development of atherosclerosis is a process that proceeds from positive remodeling to negative remodeling; negative remodeling is a late remodeling form of plaque, with a relatively stable state (41). Some lipid cores may outflow at the onset of an ischemic event due to the instability of the plaque during positive remodeling (39). Therefore, negative remodeling is more common in patients with severe symptomatic stenosis (namely late disease). Therefore, large plaque burden and positive remodeling may be the influencing factors of new cerebral infarctions in the BA territory, although there were insignificances in the multivariate analysis. The small sample size may be the cause of these results, which warrants verification through further studies with larger sample sizes.

\section{Limitation}

The current study has several limitations. First, the study data were limited due to the retrospective nature of the study. It was difficult to subdivide the smoking state of the patients into 
currently smoking and previously smoking without more details of smoking, however, it has been found that the devastating effects of tobacco smoking on vessels are lasting and do not subside easily after quitting (31). Several studies have reported better short-term outcomes in smokers, including previously and currently smoking compared with non-smokers (42). Second, the evaluation of plaque characteristics is given increasing attention nowadays. HR-MRI is an effective non-invasive method to assess plaque characteristics. However, it was not easily available for patients in our center at the early stage of the study. Third, serum biochemical tests, such as TNF- $\alpha$ levels and intra-arterial oxidative stress, and white matter damage may be related to new cerebral infarctions (43). However, the availability of these examinations was limited in the current analysis.

\section{CONCLUSION}

Few studies have investigated the risk factors for new cerebral infarctions in the BA territory after endovascular treatment for BA stenosis in Chinese individuals. The plaque characteristics are not associated with new cerebral infarctions in the distribution of BA, although a large plaque burden and positive remodeling are more likely to appear in patients with new cerebral infarctions after BA stenting, which warrants further studies with larger sample sizes. As for smoking, the inverse correlation with new cerebral infarctions in the BA territory should be verified by large-scale prospective randomized controlled trials.

\section{REFERENCES}

1. Bamford J, Sandercock P, Dennis M, Burn J, Warlow C. Classification and natural history of clinically identifiable subtypes of cerebral infarction. Lancet. (1991) 337:1521-6. doi: 10.1016/0140-6736(91)9 3206-O

2. Fiorella D, Derdeyn CP, Lynn MJ, Barnwell SL, Hoh BL, Levy EI, et al. Detailed analysis of periprocedural strokes in patients undergoing intracranial stenting in Stenting and Aggressive Medical Management for Preventing Recurrent Stroke in Intracranial Stenosis (SAMMPRIS). Stroke. (2012) 43:2682-8. doi: 10.1161/STROKEAHA.112.6 61173

3. Rothwell PM. Antiplatelet treatment to prevent early recurrent stroke. $N$ Engl J Med. (2020) 383:276-8. doi: 10.1056/NEJMe2018927

4. Faggioli G, Ferri M, Rapezzi C, Tonon C, Manzoli L, Stella A. Atherosclerotic aortic lesions increase the risk of cerebral embolism during carotid stenting in patients with complex aortic arch anatomy. J Vasc Surg. (2009) 49:805. doi: 10.1016/j.jvs.2008.08.014

5. Tedesco MM, Lee JT, Dalman RL, Lane B, Loh C, Haukoos JS, et al. Postprocedural microembolic events following carotid surgery and carotid angioplasty and stenting. J Vasc Surg. (2007) 46:24450. doi: 10.1016/j.jvs.2007.04.049

6. Pullicino P. Review: silent brain infarcts are common in the elderly general population. Evid Based Med. (2008) 13:88. doi: 10.1136/ebm.13.3.88

7. Fanning JP, Wong AA, Fraser JF. The epidemiology of silent brain infarction: a systematic review of population-based cohorts. BMC Med. (2014) 12:119. doi: 10.1186/s12916-014-0119-0

8. Smith EE, Saposnik G, Biessels GJ, Doubal FN, Fornage M, Gorelick $\mathrm{PB}$, et al. Prevention of stroke in patients with silent cerebrovascular disease: a scientific statement for healthcare professionals from the American Heart Association/American Stroke Association. Stroke. (2017) 48:e4471. doi: 10.1161/STR.0000000000000116

\section{DATA AVAILABILITY STATEMENT}

The raw data supporting the conclusions of this article will be made available by the authors, without undue reservation.

\section{ETHICS STATEMENT}

The studies involving human participants were reviewed and approved by the Ethics Committee of Xuanwu Hospital, Capital Medical University. The patients/participants provided their written informed consent to participate in this study.

\section{AUTHOR CONTRIBUTIONS}

JL contributed to the preparation of the manuscript and data collection. JL, LL, YF, and RY contributed to the data collection. $\mathrm{KY}$ and TW contributed to data analysis and interpretation. LJ, YM, PG, and BY contributed to the experimental design and manuscript revision. All authors contributed to the article and approved the submitted version.

\section{FUNDING}

This study was funded by the National Key Research and Development Program of China (2016YFC1301703) and Beijing Science and Technology Commission (D161100003816002).

9. Vermeer SE, Longstreth WT Jr., Koudstaal PJ. Silent brain infarcts: a systematic review. Lancet Neurol. (2007) 6:6119. doi: 10.1016/S1474-4422(07)70170-9

10. Zhu XJ, Wang W, Liu ZJ. High-resolution magnetic resonance vessel wall imaging for intracranial arterial stenosis. Chin Med J (Engl). (2016) 129:136370. doi: 10.4103/0366-6999.182826

11. Yu J, Li ML, Xu YY, Wu SW, Lou M, Mu XT, et al. Plaque distribution of low-grade basilar artery atherosclerosis and its clinical relevance. BMC Neurol. (2017) 17:8. doi: 10.1186/s12883-016-0785-y

12. Lou X, Ma N, Ma L, Jiang WJ. Contrast-enhanced 3T high-resolution MR imaging in symptomatic atherosclerotic basilar artery stenosis. AJNR Am J Neuroradiol. (2013) 34:513-7. doi: 10.3174/ajnr.A3241

13. Huang B, Yang WQ, Liu XT, Liu HJ, Li PJ, Lu HK. Basilar artery atherosclerotic plaques distribution in symptomatic patients: a 3.0T high-resolution MRI study. Eur J Radiol. (2013) 82:e199-203. doi: 10.1016/j.ejrad.2012.10.031

14. Samuels OB, Joseph GJ, Lynn MJ, Smith HA, Chimowitz MI. A standardized method for measuring intracranial arterial stenosis. AJNR Am J Neuroradiol. (2000) 21:643-6. Available online at: http://www.ajnr.org/content/ajnr/21/4/ 643.full.pdf

15. Shi Z, Li J, Zhao M, Peng W, Meddings Z, Jiang T, et al. Quantitative histogram analysis on intracranial atherosclerotic plaques: a highresolution magnetic resonance imaging study. Stroke. (2020) 51:2161-9. doi: 10.1161/STROKEAHA.120.029062

16. Luo J, Wang T, Gao P, Krings T, Jiao L. Endovascular treatment of intracranial atherosclerotic stenosis: current debates and future prospects. Front Neurol. (2018) 9:666. doi: 10.3389/fneur.2018.00666

17. Gao P, Zhao Z, Wang D, Wu J, Cai Y, Li T, et al. China Angioplasty and Stenting for Symptomatic Intracranial Severe Stenosis (CASSISS): a new, prospective, multicenter, randomized controlled trial in China. Interv Neuroradiol. (2015) 21:196-204. doi: 10.1177/1591019915581778

18. Jia B, Liebeskind DS, Ma N, Gao F, Mo D, Luo G, et al. Factors associated with perforator stroke after selective basilar artery angioplasty or stenting. 
J Neurointerv Surg. (2017) 9:738-42. doi: 10.1136/neurintsurg-2016-0 12329

19. Zaidat OO, Castonguay AC, Fitzsimmons BF, Woodward BK, Wang Z, Killer-Oberpfalzer $M$, et al. Design of the Vitesse Intracranial Stent Study for Ischemic Therapy (VISSIT) trial in symptomatic intracranial stenosis. J Stroke Cerebrovasc Dis. (2013) 22:1131-9. doi: 10.1016/j.jstrokecerebrovasdis.2012.10.021

20. Zaidat OO, Fitzsimmons BF, Woodward BK, Wang Z, Killer-Oberpfalzer M, Wakhloo A, et al. Effect of a balloon-expandable intracranial stent vs medical therapy on risk of stroke in patients with symptomatic intracranial stenosis: the VISSIT randomized clinical trial. JAMA. (2015) 313:12408. doi: $10.1001 /$ jama.2015.1693

21. Masuda J, Nabika T, Notsu Y. Silent stroke: pathogenesis, genetic factors and clinical implications as a risk factor. Curr Opin Neurol. (2001) 14:7782. doi: 10.1097/00019052-200102000-00012

22. Saavedra Perez HC, Direk N, Hofman A, Vernooij MW, Tiemeier H, Ikram MA. Silent brain infarcts: a cause of depression in the elderly? Psychiatry Res. (2013) 211:180-2. doi: 10.1016/j.pscychresns.2012.06.005

23. Longstreth WT Jr., Dulberg C, Manolio TA, Lewis MR, Beauchamp NJ Jr., O'Leary D, et al. Incidence, manifestations, and predictors of brain infarcts defined by serial cranial magnetic resonance imaging in the elderly: the Cardiovascular Health Study. Stroke. (2002) 33:237682. doi: 10.1161/01.STR.0000032241.58727.49

24. Wu C, Li C, Zhao W, Xie N, Yan F, Lian Y, et al. Elevated trimethylamine noxide related to ischemic brain lesions after carotid artery stenting. Neurology. (2018) 90:e1283-90. doi: 10.1212/WNL.0000000000005298

25. Samaniego EA, Shaban A, Ortega-Gutierrez S, Roa JA, Hasan DM, Derdeyn C, et al. Stroke mechanisms and outcomes of isolated symptomatic basilar artery stenosis. Stroke Vasc Neurol. (2019) 4:189-97. doi: 10.1136/svn-2019-000246

26. Jha P. Avoidable global cancer deaths and total deaths from smoking. Nat Rev Cancer. (2009) 9:655-64. doi: 10.1038/nrc2703

27. Conroy RM, Pyörälä K, Fitzgerald AP, Sans S, Menotti A, De Backer G, et al. Estimation of ten-year risk of fatal cardiovascular disease in Europe: the SCORE project. Eur Heart J. (2003) 24:987-1003. doi: 10.1016/S0195-668X(03)00114-3

28. Erhardt L. Cigarette smoking: an undertreated risk factor for cardiovascular disease. Atherosclerosis. (2009) 205:2332. doi: 10.1016/j.atherosclerosis.2009.01.007

29. Sunyer J, Forastiere F, Pekkanen J, Plana E, Kolz M, Pistelli R, et al. Interaction between smoking and the interleukin-6 gene affects systemic levels of inflammatory biomarkers. Nicotine Tob Res. (2009) 11:134753. doi: $10.1093 / \mathrm{ntr} / \mathrm{ntp} 144$

30. Barbash GI, Reiner J, White HD, Wilcox RG, Armstrong PW, Sadowski $Z$, et al. Evaluation of paradoxic beneficial effects of smoking in patients receiving thrombolytic therapy for acute myocardial infarction: mechanism of the "smoker's paradox" from the GUSTO-I trial, with angiographic insights. Global Utilization of Streptokinase and Tissue-Plasminogen Activator for Occluded Coronary Arteries. J Am Coll Cardiol. (1995) 26:12229. doi: 10.1016/0735-1097(95)00299-5

31. Ramotowski B, Gurbel PA, Tantry U, Budaj A. Smoking and cardiovascular diseases: paradox greater than expected? Pol Arch Intern Med. (2019) 129:7006. doi: 10.20452/pamw.14931

32. Desai NR, Mega JL, Jiang S, Cannon CP, Sabatine MS. Interaction between cigarette smoking and clinical benefit of clopidogrel. J Am Coll Cardiol. (2009) 53:1273-8. doi: 10.1016/j.jacc.2008.12.044
33. Doig D, Turner EL, Dobson J, Featherstone RL, Lo RT, Gaines PA, et al. Predictors of stroke, myocardial infarction or death within 30 days of carotid artery stenting: results from the international carotid stenting study. Eur J Vasc Endovasc Surg. (2016) 51:327-34. doi: 10.1016/j.ejvs.2015.08.013

34. Gremmel T, Steiner S, Seidinger D, Koppensteiner R, Panzer S, Kopp CW. Smoking promotes clopidogrel-mediated platelet inhibition in patients receiving dual antiplatelet therapy. Thromb Res. (2009) 124:588-91. doi: 10.1016/j.thromres.2009.06.012

35. Bliden KP, Dichiara J, Lawal L, Singla A, Antonino MJ, Baker $\mathrm{BA}$, et al. The association of cigarette smoking with enhanced platelet inhibition by clopidogrel. J Am Coll Cardiol. (2008) 52:531-3. doi: 10.1016/j.jacc.2008.04.045

36. Liu Z, Xiang Q, Mu G, Xie Q, Zhou S, Wang Z, et al. The effect of smoking on residual platelet reactivity to clopidogrel: a systematic review and metaanalysis. Platelets. (2020) 31:3-14. doi: 10.1080/09537104.2019.1572878

37. Cao Y, Sun Y, Zhou B, Zhao H, Zhu Y, Xu J, et al. Atherosclerotic plaque burden of middle cerebral artery and extracranial carotid artery characterized by MRI in patients with acute ischemic stroke in China: association and clinical relevance. Neurol Res. (2017) 39:344-50. doi: 10.1080/01616412.2017.1281196

38. Ma N, Jiang WJ, Lou X, Ma L, Du B, Cai JF, et al. Arterial remodeling of advanced basilar atherosclerosis: a 3-tesla MRI study. Neurology. (2010) 75:253-8. doi: 10.1212/WNL.0b013e3181e8e714

39. Feng C, Hua T, Xu Y, Liu XY, Huang J. Arterial remodeling of basilar atherosclerosis in isolated pontine infarction. Neurol Sci. (2015) 36:54751. doi: 10.1007/s10072-014-1994-Z

40. Li F, Chen QX, Chen ZB, Tian DF, Cai Q. Magnetic resonance imaging of plaque burden in vascular walls of the middle cerebral artery correlates with cerebral infarction. Curr Neurovasc Res. (2016) 13:26370. doi: 10.2174/1567202613666160829095324

41. Roth W, Morgello S, Goldman J, Mohr JP, Elkind MS, Marshall RS, et al. Histopathological differences between the anterior and posterior brain arteries as a function of aging. Stroke. (2017) 48:638-44. doi: 10.1161/STROKEAHA.116.015630

42. Berger JS, Bhatt DL, Steinhubl SR, Shao $M$, Steg PG, Montalescot G, et al. Smoking, clopidogrel, and mortality in patients with established cardiovascular disease. Circulation. (2009) 120:2337-44. doi: 10.1161/CIRCULATIONAHA.109.8 66533

43. Lin C, Tang X, Shi Z, Zhang L, Yan D, Niu C, et al. Serum tumor necrosis factor $\alpha$ levels are associated with new ischemic brain lesions after carotid artery stenting. J Vasc Surg. (2018) 68:771-8. doi: 10.1016/j.jvs.2017. 11.085

Conflict of Interest: The authors declare that the research was conducted in the absence of any commercial or financial relationships that could be construed as a potential conflict of interest.

Copyright (c) 2021 Luo, Li, Wang, Yang, Feng, Yang, Ma, Gao, Yang and Jiao. This is an open-access article distributed under the terms of the Creative Commons Attribution License (CC BY). The use, distribution or reproduction in other forums is permitted, provided the original author(s) and the copyright owner(s) are credited and that the original publication in this journal is cited, in accordance with accepted academic practice. No use, distribution or reproduction is permitted which does not comply with these terms. 